\title{
ENSEÑAR MATEMATICA FINANCIERA EN LA UNIVERSIDAD
}

\author{
FINANCIAL MATHEMATICS TEACHING IN THE UNIVERSITY \\ Manuel Alberto Hidalgo Tupia* \\ Docente Auxiliar de la Facultad de Ciencias Contables \\ Universidad Nacional Mayor de San Marcos-UNMSM / Lima-Perú \\ [Recepción: Abril 2016/ Conformidad: Mayo 2016]
}

\section{RESUMEN}

El presente artículo constituye la presentación de los resultados de la investigación titulada "Efectos de la aplicación de una metodología de enseñanza activa, pertinente y heurística en el aprendizaje de la matemática financiera", desarrollada por el autor para obtener el grado académico de Doctor en Educación, sustentada en noviembre de 2015, en la Unidad de Posgrado de la Facultad de Educación de la Universidad Nacional Mayor de San Marcos.

La investigación consistió en el estudio de los fundamentos de la pedagogía aplicada a la enseñanza de las matemáticas, y como se utiliza para el caso de las matemáticas aplicadas, específicamente, matemáticas financieras. Se buscó dentro del enfoque teórico existente las características deseables de una metodología de enseñanza de la matemática financiera, formulándose con esa base una metodología de enseñanza basada con las características de activismo, pertinencia, y heurística, la cual fue aplicada en la enseñanza del curso en una universidad de Lima, para medir posteriormente, su efecto en el aprendizaje de los alumnos. Se aplicó el tratamiento a un grupo experimental y se registró también a los resultados de un grupo control, en el transcurso de dos años. Se aplicó una prueba estadística de la hipótesis, cuyos resultados que probaron la misma, han demostrado el efecto de metodología de enseñanza, la cual fue descripta ampliamente en sus fundamentos, condiciones, estrategias, planificación de sesiones de aprendizaje, materiales y evaluación.

\section{Palabras clave:}

Pedagogía; enseñanza; didáctica; aprendizaje; matemática; matemática financiera; activismo; pertinencia; heurística.

\begin{abstract}
This article presents the results of the research entitled "Application effects of an active, relevant and heuristic methodology in the learning of mathematical finance" developed by the author for obtaining the degree of Doctor in Education, defended on November 2015, in the Graduate School of Education of the Universidad Nacional Mayor de San Marcos.

The research involved the study of the pedagogy's fundamentals applied to the teaching of mathematics, like in the case of applied mathematics, specifically, financial mathematics. The desirable features of a teaching methodology for financial mathematics were sought, within the existing theoretical approach. On that base, a teaching methodology was formulated, centered on the characteristics of activism, relevance, and heuristics; and then applied in teaching course in the Universidad de Lima, to finally measure the effect on student learning. The treatment was applied to an experimental group and also to a control group, in the course of two years, plus the results were recorded. The hypothesis was statistically tested and the results support it. They show the effect of the teaching methodology, which was widely described in its foundations, conditions, strategies, planning training sessions, materials and evaluation.
\end{abstract}

\section{Keywords:}

Pedagogy; teaching; didactics; learning; math; financial mathematics; activism; relevance; heuristics.

\footnotetext{
* Magíster en Educación. Economista. Email: mhidalgot@unmsm.edu.pe
} 


\section{ANTECEDENTES}

La problemática educativa en el país, que es común a muchos países del mundo en cuanto al insuficiente logro de la calidad y las competencias requeridas, es difundida y ha sido conocida por el autor en su experiencia de 13 años de trabajo en la educación superior, en la Facultad de Ciencias Contables y en el sistema universitario. En el caso específico de la asignatura de Matemática Financiera en el programa de formación de adultos EPEL (Programa de Formación Profesional por Experiencia Laboral) de una universidad de Lima, el índice de desaprobación en la asignatura es mayor que los que corresponden a las demás asignaturas, y comparable con los índices de los demás cursos de Matemática.

Las causas del problema se deben buscar en las falencias de la educación básica, pero es deber del docente superar la falta de conocimientos y habilidad matemática, y su consecuencia en el mal logro de las competencias de la asignatura, para lo cual, en base a la experiencia docente acumulada, se formuló una metodología de enseñanza activa, pertinente y Heurística en el Aprendizaje de la Matemática Financiera (MEAPH), la cual posee unos fundamentos conceptuales y pedagógicos, una programación de los contenidos y una planificación de las sesiones de clase y de los materiales respectivos. Estafue validada por los expertos en su coherencia y sustento conceptual; y constituye el aporte creativo e innovador de esta investigación, al punto que merece un tratamiento más amplio en otro artículo.
Dicha metodología aparece en el informe de tesis que estará disponible próximamente en el repositorio digital de tesis de la Universidad Nacional Mayor de San Marcos.

Antes de describir brevemente esta metodología, cabe señalar que se aplicó durante un lapso de 2 años (entre 2013 y 2014) a un total de 228 alumnos del grupo experimental y 113 alumnos del grupo control de diferentes grupos - clase. Por otro lado, también se planteó una prueba de hipótesis estadística de diferencia de medias entre el grupo experimental (al cual se aplicó la metodología) y al grupo de control (al cual no se aplicó la metodología, sino la enseñanza convencional). El instrumento fue un test que mide el conocimiento conceptual y procedimental

\section{METODOLOGÍA DE ENSEÑANZA ACTIVA, PERTINENTE Y HEURÍSTICA PARA EL APRENDIZAJE DE LA MATEMÁTICA FINANCIERA}

La didáctica de las matemáticas es una disciplina en pleno desarrollo dentro de la propia didáctica, cuyos conceptos son aplicables a las matemáticas financieras, las cuales poseen, sin embargo, determinadas características específicas.

En el gráfico que sigue se resumen los elementos del activismo (A), pertinencia $(\mathrm{P})$ y heurística $(\mathrm{H})$, los cuales habiendo sido validados por la pedagogía, como se muestra en el amplia fundamentación hecha en el informe de investigación, son aplicables, en nuestro caso, a las matemáticas financieras. 
Figura No 01:

Elementos de activismo, pertinencia y heurística aplicables a la enseñanza de la matemática financiera

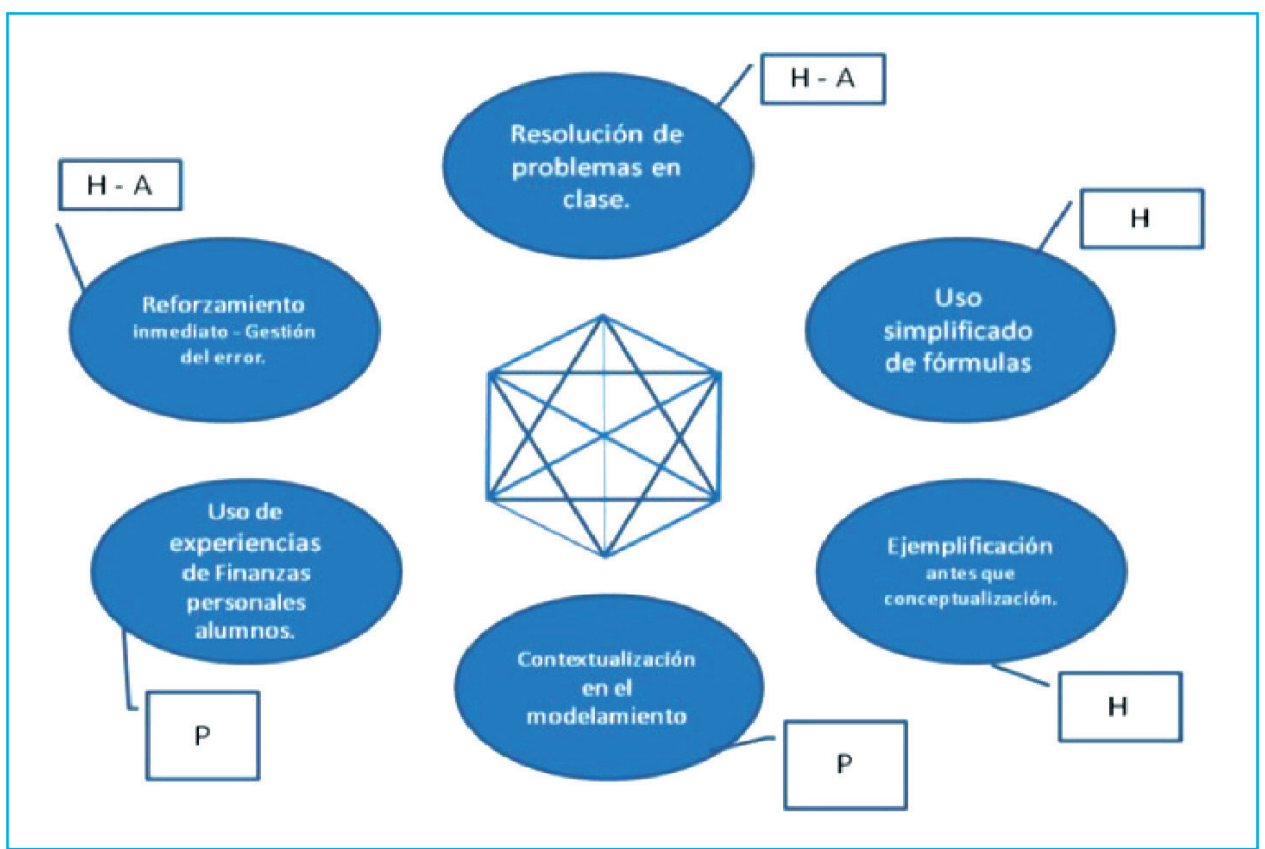

Fuente: Elaboración propia.

En síntesis, la metodología EPH desarrolla los siguientes elementos completamente interrelacionados:

\section{Uso simplificado de fórmulas}

Solo basta discutir la fórmula una vez y alentar a los alumnos a usar las consabidas propiedades de las ecuaciones para "despejar" la segunda fórmula a partir de la primera. Omitir fórmulas repetitivas o cuyas variables sean equívocas.

2. Trabajo práctico en aula de los alumnos, individual y grupal, a través de ejercicios y problemas propuestos los cuales deben ser resueltos por el docente en la pizarra para poder tener un reforzamiento inmediato. En el trabajo práctico en el aula el docente debe absolver preguntas y dificultades de comprensión, de modelamiento, de uso de la calculadora, etc.

3. Reforzamiento Inmediato, según el concepto de la psicología conductista, la cual consiste en este caso en la validación inmediata en clase del trabajo académico del alumno, en forma de casos y ejercicios propuestos.
4. Ejemplificación para pasar de lo abstracto a lo concreto.

5. Uso de las experiencias personales de los alumnos para ejemplificar las situaciones financieras.

6. Contextualización en el modelamiento.

Especialmente, el contexto que se da es empresarial y en el marco del sistema financiero peruano. Brindar el contexto permite y facilita mucho el indispensable proceso de abstracción, que se aminora con el recurso al contexto que es conocido por los estudiantes, pues se trata de alumnos que poseen experiencia laboral.

Como sostiene el autor (Hidalgo, 2016)

Se procura relacionar los conceptos financieros con las propias finanzas personales de los estudiantes (que son personas que trabajan, todos), o las aplicaciones financieras en sus centros de trabajo, como sería un préstamo corporativo. ¿Quién no ha requerido un préstamo bancario en alguna ocasión? ¿No es verdad que a mayores plazos de repago mayor será el interés pagado? En tal caso, el estudio de las finanzas es más significativo. 
En adición a lo anterior, consideran los siguientes aspectos concomitantes relacionados a estos:

\section{Gestión del error}

Si bien se ha dicho que el error no es equivocarse, el error enseña. Por ello, es muy importante gestionar el error, es decir, identificar, corregir y analizar su origen. Esta es la principal ventaja de la retroalimentación o reforzamiento inmediato, que se refiere al trabajo práctico en clase y en base al activismo. Es decir, si un alumno se equivoca en clase, eso es muy bueno, pues permitirá al docente y a los condiscípulos hacerle ver su error y corregirlo, marcando más el aprendizaje.

\section{Materiales de estudio}

Se acompaña todo con un texto autoinstuctivo, cuidadosamente preparado por el autor y docente del curso.

\section{Enfoque a la competencia procedimental}

No se puede demostrar la competencia conceptual sin demostrar la competencia procedimental.Se debe prevenir la no comprensión o los errores en el modelamiento y su aplicación, con el reforzamiento inmediato se aclaran en el mismo salón, por parte del docente o por parte de los propios compañeros, las causas de por qué al alumno "no le salió el resultado del problema”. Así, se previene que un error de comprensión se reproduzca, tanto en el modelamiento como en la aplicación.

En adición a ello, se establecieron 4 estrategias generales, subsumidas en los elementos citados anteriormente que se resumen en la siguiente figura:

Figura No 02:

Estrategias generales de la metodología EPH

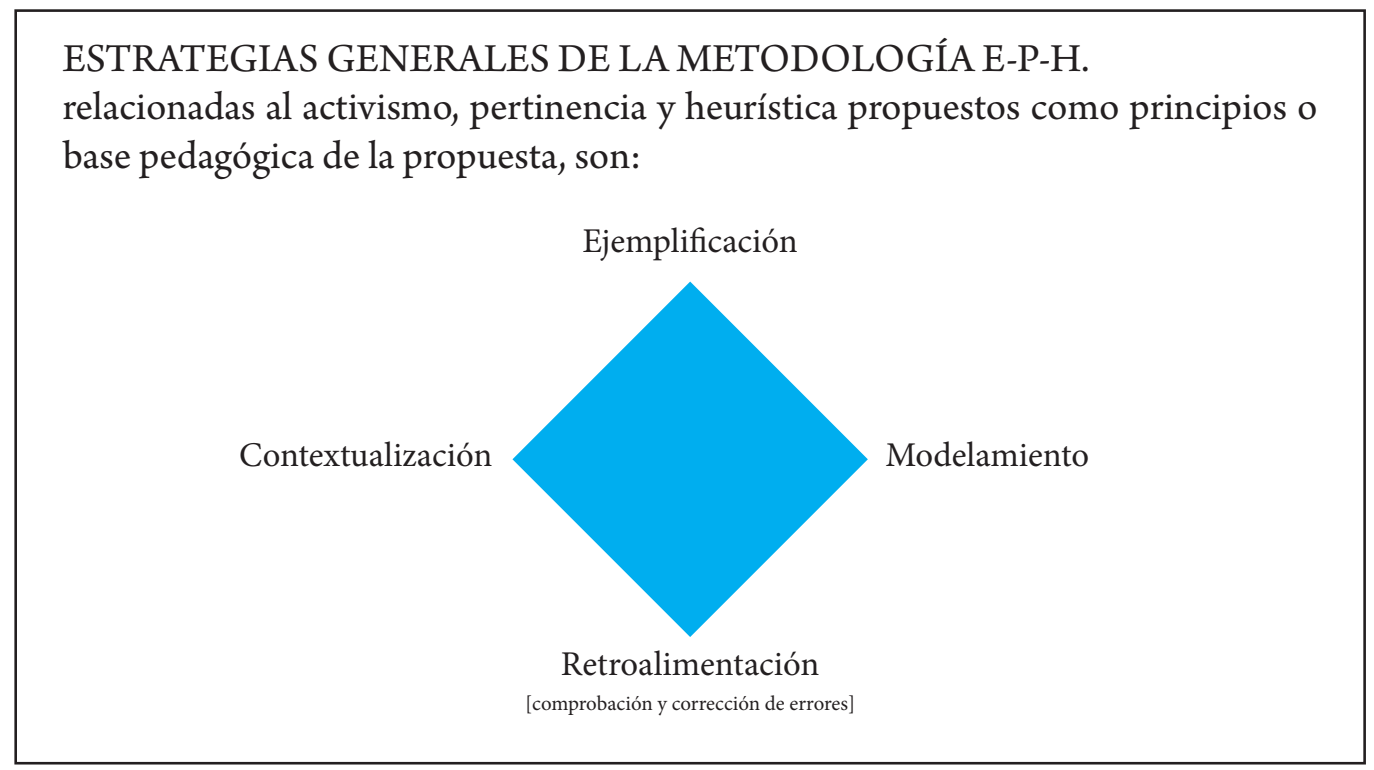

Fuente: Elaboración propia.

\section{RESULTADOS}

La metodología APH se aplicó durante un lapso de 2 años (entre 2013 y 2014) a un total de 228 alumnos del grupo experimental y 113 alumnos del grupo control (al cual no se aplicó la metodología) de diferentes grupos - clase, midiéndose los resultados en el aprendizaje en ambos casos y planteándose una prueba de hipótesis estadística de diferencia de me- dias entre el grupo experimental (al cual se aplicó la metodología) y al grupo Control (al cual no se aplicó la metodología, sino la enseñanza convencional). El instrumento fue un test que medía el conocimiento conceptual y procedimental.

En forma de resumen conclusivo, se muestra la siguiente figura que expone la diferencia de las medias, tal como sigue:

32/ QUIPURAMAYOC | Vol. 24(45 A) 2016 
Figura No 03:

Rendimiento de grupos control y experimental.

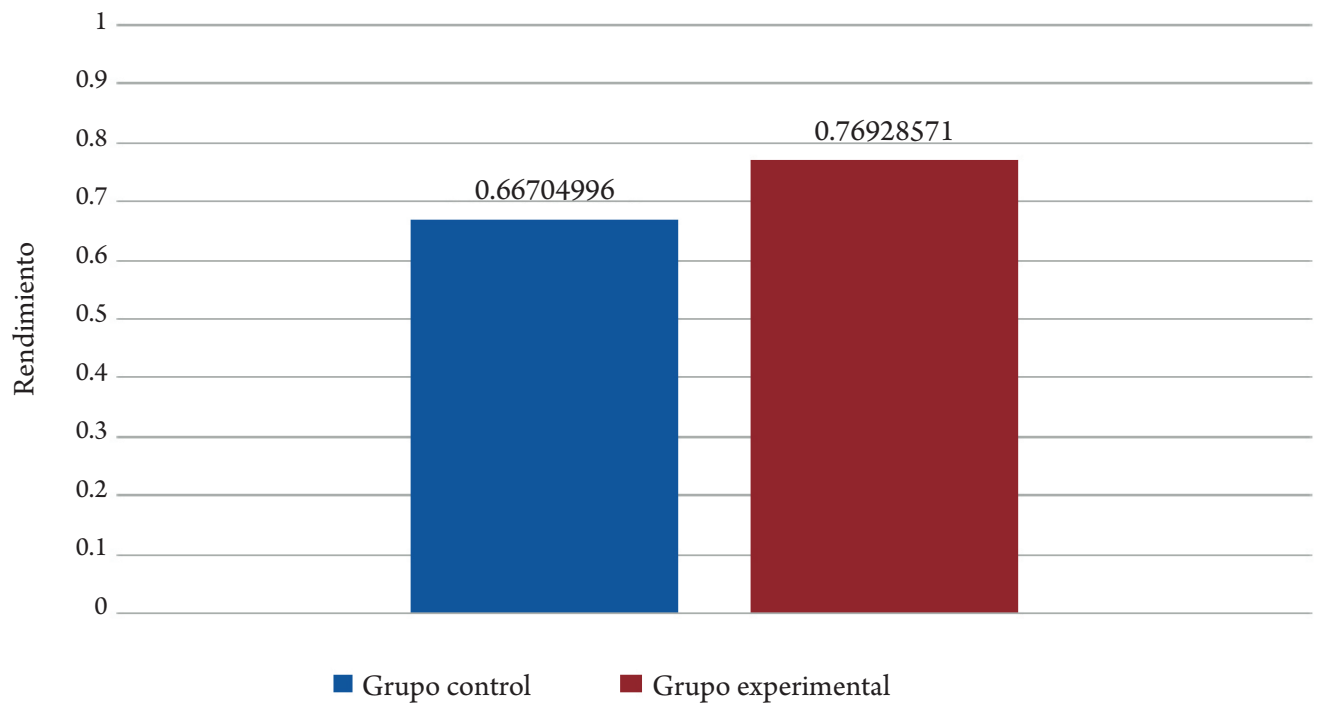

Fuente: Elaboración propia.

Através de estos gráficos se probó que las muestras eran normales, tal como se observa en adición en las figuras que siguen(Figura No 04 y Figura No 05). A continuación, se muestran los resultados de la primera de ellas respecto al grupo control:

Figura No 04:

Distribución de frecuencia para grupo control.

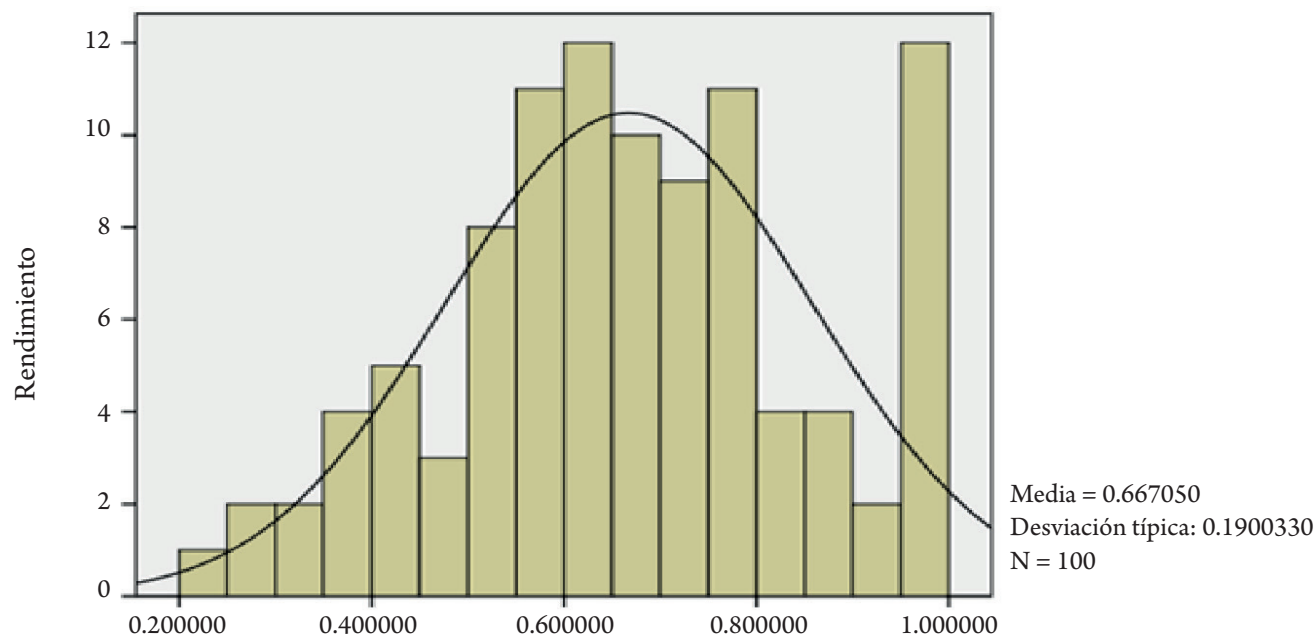

Fuente: Elaboración propia.

Asimismo, se presenta la correspondiente al grupo experimental: 
Figura No 05:

Distribución de frecuencia para grupo experimental.

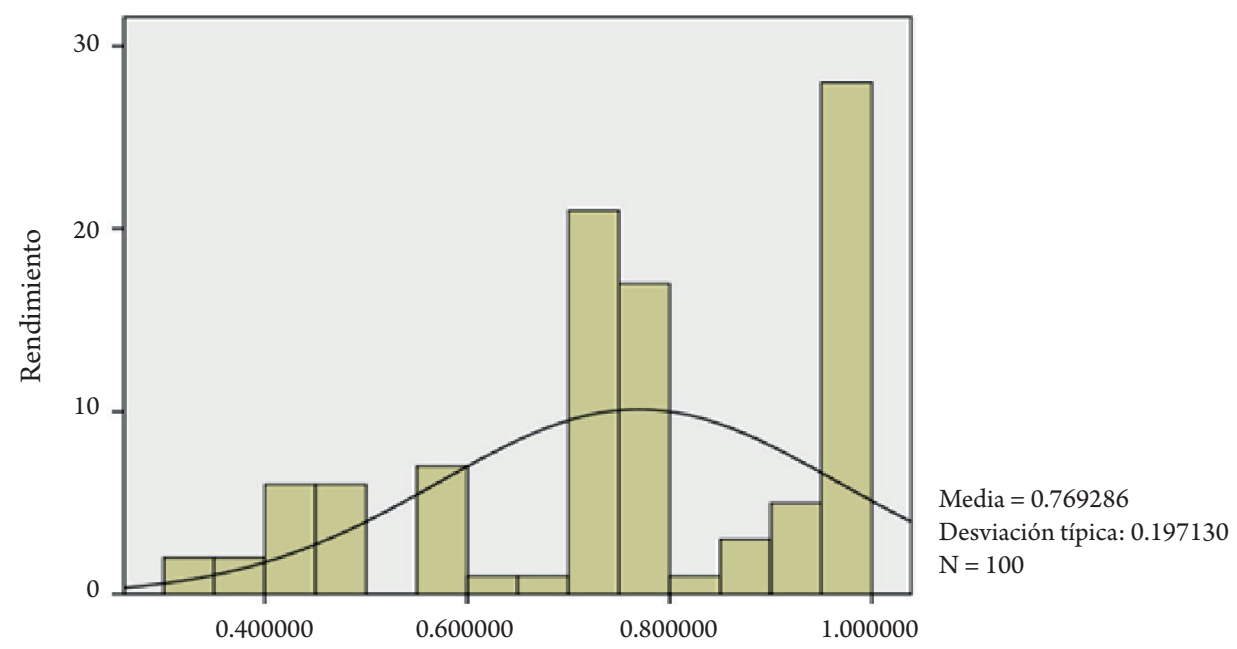

Fuente: Elaboración propia.

Se aplicó la prueba "t" de Students, la cual arrojó como resultados 0.667049956 puntos para el índice de la prueba en grupo control (X), siendo este menor que los 0.769285714 puntos para el índice de la prueba en grupo experimental (Y). De este modo, se determinó, con un nivel de significación cercano al $100 \%$, que existe una diferencia estadísticamente significativa en el rendimiento de los alumnos entre el grupo experimental y el grupo control, favorable al primero.

En palabras más familiares, mientras que los estudiantes que no recibieron la metodología obtuvieron un calificativo de 13.34 en calificación vigesimal, quienes recibieron la metodología obtuvieron un 15.38 , siendo la diferencia no atribuible a otro factor externo, los cuales fueron controlados.

Según la regla de decisión por lo cual, dada la diferencia de medias y una medida de significación menor al 0,05, se daba por rechazada la hipótesis nula $\mathrm{H} 0$ y por lo cual se da por aceptada la hipótesis alterna (H1), que es la hipótesis de la investigación, se cumple $\mathrm{H} 1$ (Hipótesis alterna) la cual supone que existe diferencia estadísticamente significativa en el rendimiento de los alumnos entre el Grupo experimental y el Grupo Control, favorable al primero.

\section{CONCLUSIONES}

La conclusión general fue que la estrategia didáctica activa, pertinente y heurística de enseñanza de la matemática financiera tuvo un impacto significativo y positivo en el rendimiento de los estudiantes participantes en el Curso de Matemática Financiera, en el tema representativo de Anualidades.

Se recomienda publicar el informe de investigación (o una síntesis de él) y sensibilizar a la comunidad docente y profesional sobre la problemática planteada y los rumbos de solución. En adición, se cree conveniente difundir en la comunidad docente los conceptos de la estrategia didáctica difundiendo los principios y estrategias de la metodología didáctica activa, pertinente y heurística que han sido propuestos.

Las limitaciones encontradas para la investigación fueron el enfoque de la investigación a una modalidad especifica de organización escolar (programa dirigido a personas que trabajan, en un formato muy limitado de tiempo), lo cual limita la generalización de las conclusiones y la imposibilidad de aplicar una pre prueba por razones de programación, esto último impide asegurar más el aislamiento de las variables extrañas. 
En consideración de lo anterior, se recomienda ampliar la investigación con mayor número de pruebas a otros aspectos del curso de Matemática Financiera y a otros grupos clase de otras universidades del país y del extranjero en las carreras de las ciencias empresariales tanto en la modalidad semipresencial como presencial, para así ampliar la generalización de las conclusiones de la investigación reseñada.

\section{REFERENCIAS BIBLIOGRÁFICAS}

1. Tesis: "Efectos de la Aplicación de una Metodología de Enseñanza Activa, Pertinente y Heurística en el Aprendizaje de la Matemática Financiera”, para Optar el Grado de: Doctor en Educación por Manuel Alberto Hidalgo Tupia. 2015

2. Manuel Alberto Hidalgo Tupia:Efectos de la Aplicación de una Metodología de Enseñanza Activa, Pertinente y Heurística en el Aprendizaje de la Matemática Financiera”, articulo remitido y aceptado por la Revista "Calidad y Creatividad", Universidad de Ricardo Palma, Lima. 2016.

3. ARY, D, CHESER, L., y RAZAVIEH, A. (1989). Introducción a la Investigación pedagógica. México: Mc Graw-Hill Interamericana.

4. D’AMORE, B. (2006). Didáctica de las matemáticas. Bogotá. Cooperativa Editorial Magisterio.

5. GARCÍA, A y otros. (2012). La enseñanza de la Matemática Financiera. Un modelo didáctico mediado por TIC. Biblioteca Virtual de Derecho, Ciencia Política y Economía. Recuperado el 15/08/2012 de:http://www.eumed.net/ libros/2010f/867/indice.htm.

6. LÁZARO, D. (2012). Estrategias Didácticas y Aprendizaje de la Matemática en el Programa de Estudios por Experiencia Laboral. Universidad de San Martin de Porras. Instituto para la Calidad de la Educación Sección de Posgrado. Lima, Perú. Recuperado el 14 de enero de 2014, en: http:// www.repositorioacademico.usmp.edu.pe/bitstream/usmp/613/3/lazaro_db.pdf

7. ROQUE, J. (2012). Influencia de la Enseñanza de la Matemática Basada en la Resolución de Problemas en el Mejoramiento del Rendimiento Académico. El Caso de los Ingresantes a la Escuela de Enfermería de la Universidad Alas Peruanas 2008-I. Universidad San Martin de Porres. Recuperado el 11 de enero de 2014 en: http:// cybertesis.unmsm.edu.pe/bitstream/cybertesis/1704/1/roque_sj.pdf

8. ROSS, S, R. WESTFIELD y J. JAFFE. (2012). Finanzas corporativas. Ed. Mc Graw Hill. 9a Edición. México D.F

9. SIFUENTES, M. (2013). Organizadores Gráficos y Medios Audiovisuales en el Aprendizaje Significativo de los Estudiantes en el Área de Ciencias Sociales del CEBA Ventura Ccalamaqui de Barranca durante el 2012. Artículo en Revista "Logos". Facultad de Ciencias Empresariales. Universidad Alas Peruanas. Año VI - 2013 - Vol. No 7. Páginas $87-104$. 